\title{
Rendimiento académico en estudiantes de secundaria según asignaturas, estrato socioeconómico y su contacto con el conflicto armado en Colombia
}

\author{
Academic performance in high school students \\ according to study courses, socioeconomic strata and \\ their contact with the armed conflict in Colombia
}

Magda J. Rojas Bahamón. * María L. López de Parra. ${ }^{\odot}$ Diego F. Arbeláez Campillo" y Lucelly Correa Cruz»

\section{RESUMEN}

Este documento pretende hacer la descripción del rendimiento académico de los estudiantes de educación básica y media de la Institución Educativa Antonio Ricaurte (IEAR) de Florencia, departamento del Caquetá, según las asignaturas básicas establecidas en la Ley General de Educación Colombiana (Ley 115 de 1994), su estrato socioeconómico, su género y su contacto con el conflicto armado. El estudio está enmarcado en el proyecto de investigación "Representaciones sociales de los estudiantes de la IEAR acerca del postconflicto" del programa ONDAS Colciencias, el cual concibe en unos de sus interrogantes, indagar si el conflicto armado incide en el desempeño académico de los estudiantes. Desde el ámbito metodológico se realizaron análisis estadísticos descriptivos y correlacionales de las variables. La transcendencia del estudio se valora en los aportes que el proceso estadístico hizo en torno al análisis de la información del desempeño escolar de los estudiantes en función de la variable conflicto armado, que en el departamento del Caquetá ha tenido mucha influencia en el ámbito social de los habitantes.

Palabras clave: análisis correlacional, desempeño escolar, conflicto armado, Institución educativa Antonio Ricaurte

\section{ABSTRACT}

This document aims to make the description of the academic performance of students in primary and secondary education in the Institute Antonio Ricaurte (IEAR) in Florencia, Caqueta, according to the basic subjects established in the General Law of Colombian Education (Act 115 of 1994 ), their socio-economic status, gender and contact with the armed conflict. The study is framed in the research project "Social representations of students of IEAR about the post-conflict" the ONDAS Colciencias program, which conceived in one of its questions, inquire whether the armed conflict affects the academic performance of students. From the methodological field, descriptive statistical analysis and correlational variables were performed. The significance of the study is assessed on the contributions that statistical process made around the analysis of information school student performance based on the variable armed conflict, in Caquetá has been very influential in the social behaviour of their habitants.

Key words: correlational analysis, school performance, armed conflict, Educational Institution Antonio Ricaurte

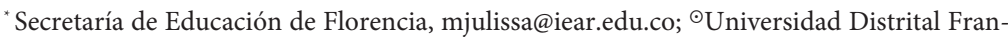
cisco José de Caldas, mar.lopez@udla.edu.co; -Facultad de Ciencias Contables Económicas y Administrativa de la Universidad de la Amazonia, Florencia, Caquetá, dfaca@hotmail.com; $\diamond$ Facultad de Ciencias Contables Económicas y Administrativas, Universidad de la Amazonia, Florencia, Caquetá, Colombia; 1.correa@udla.edu.co.
} 


\section{Introducción}

Colombia ha sido un país azotado por la violencia desde hace muchos ańos y se ha visto envuelto en problemas sociales relacionados con la existencia de grupos al margen de la ley.

El Caquetá principalmente ha sido epicentro/cuna de grupos terroristas desde la década de los sesenta; ha sido escenario de conflictos y procesos de paz fallidos, que la población ha tenido que soportar a lo largo de muchos ańos. Por esa razón, muchos pobladores del Caquetá han crecido en contextos de constante violencia en la que los secuestros, el desplazamiento forzado, la extorsión, el reclutamiento de menores, las muertes violentas, entre otras, hacen parte del diario vivir, a tal punto, que las personas se insensibilizan frente a la situación y parece no importarles la desgracia del otro.

En el Caquetá existen estudios de caso que han concluido que el tema de violencia, exactamente el que tiene que ver con el conflicto armado, agrava la salud mental en el departamento (Organización Médicos Sin Fronteras -MSF-, 2009) y han establecido que los pobladores de esta zona del país sufren de un trastorno adaptativo, expresado en el deterioro significativo de la actividad social o laboral, producto de un clima que los investigadores llaman "estresante".

De esta manera. este trastorno adaptativo se ve evidenciado, de alguna manera, en el comportamiento de muchas personas en la sociedad caqueteña y florenciana, no solamente constituye en sí mismo un factor de riesgo para la salud mental de las personas directamente expuestas, sino que además genera condiciones en las cuales emergen otros factores de riesgo que también contribuyen al deterioro de la salud y la calidad de vida de las personas y las comunidades.

Según la UNESCO (2011), los conflictos armados siguen representando un importante obstáculo para el desarrollo humano en muchas partes del mundo, pero hasta ahora se han ignorado ampliamente sus repercusiones en la educación. Asimismo, Sánchez y Díaz (2005) sostienen que el impacto de la intensidad del conflicto armado colombiano sobre el desarrollo social no ha sido cuantificado rigurosamente. 
De igual manera, aunque existen investigaciones (González, 2003; Gómez, 2003; Reinoso, 2011) que abordan el estudio de factores que inciden en el desempeño escolar como motivación, inteligencia y aptitudes, autoconcepto, estrategias y estilos de aprendizaje, hábitos de estudio y aspectos familiares, para muchos académicos colombianos el Estado debe considerar que la crisis de la educación en Colombia obedece, en gran medida, a factores diferentes a los didácticos o curriculares. En este sentido. Avila, Quiñonez y otros (2014) manifiestan que las causas que ocasionan la crisis educativa tienen profundas raíces en la convergencia de dos situaciones nefastas: el contexto social y económico del país, y la equivocada aplicación de las políticas educativas neoliberales.

En Florencia y Caquetá han sido escasos los estudios que aborden elementos de conflicto armado y educación. Cruz (2000) presenta un referente importante en este campo mediante un diagnóstico sobre la presencia de conflictos y su tratamiento en las instituciones educativas del municipio, y determina que existe una relación indisoluble entre formación y prácticas de convivencia pacífica, aspecto que incide directamente en el desempeño escolar.

Por lo anterior, es indudable indicar que en el desempeño académico intervienen otros factores de tipo social, razón por la cual es necesario ahondar en estudios que permitan determinar la incidencia de este tipo de factores en el desempeño académico de los estudiantes, puesto que los informes que se encuentran acerca de desempeño escolar, se orientan a mostrar rankins de instituciones educativas del municipio en función de las pruebas externas como SABER 5, SABER 9 y SABER 11.

En la Institución Educativa Antonio Ricaurte de Florencia existen muchos estudiantes que han estado expuestos a escenarios violentos o tienen familiares que lo han estado. Estos episodios de violencia hacen que los jóvenes se formen en ambientes poco tolerantes, aspectos que se ven evidenciados en las rińas constantes en clase, la burla hacia el otro o la respuesta agresiva frente a ciertos comentarios de grupo.

En razón a lo anterior, este estudio plantea dos objetivos: a) describir el rendimiento académico de los estudiantes de la 
Institución Educativa Antonio Ricaurte de Florencia según las asignaturas básicas establecidas en la Ley General de Educación Colombiana (Ley 115 de 1994), su estrato socioeconómico, sexo y b) Determinar la incidencia de la variable conflicto armado en el rendimiento académico de los estudiantes de la IEAR.

Para lo anterior, el estudio abordó un análisis correlacional. Se inició con un proceso descriptivo de las variables con base en su resumen univariado, las características de correlación entre la variable "asignaturas" $y$, finalmente, los procedimientos correlacionales entre variables.

En los resultados del proceso estadístico se obtuvo información muy valiosa acerca de la incidencia negativa de la variable "conflicto armado" sobre el rendimiento académico de los estudiantes de la IEAR de Florencia, así como indicadores que evidencian que las asignaturas que involucran actividades deportivas, culturales, artísticas, pueden generar mejoras sustanciales en los resultados académicos de estudiantes, a pesar de haber vivido situaciones de conflicto armado.

\section{Método}

\section{Población y descripción de los datos}

El estudio se realizó en la Institución Educativa Antonio Ricaurte (IEAR) de Florencia, Caquetá, con una población compuesta por 1620 estudiantes que culminaron satisfactoriamente primer periodo académico 2015.

La tabla de datos se obtuvo con una consulta realizada en MySQL al sistema de infomación académico de la IEAR, en la que se obtuvo información del desempeño escolar de los estudiantes durante el primer periodo en las asignaturas básicas establecidas por la Ley General de Educación de Colombia: ciencias naturales, ciencias sociales, educación artística, educación ética, educación física, educación religiosa, lengua castellana, idioma extranjero (inglés), tecnología e informática y matemáticas. Asimismo, se hizo la consulta del estrato social del estudiante, la edad, el sexo, el municipio de procedencia y si era víctima del conflicto. Cabe aclarar que la información de la condición del estudiante como 
víctima de conflicto armado se registra en el sistema de información, de acuerdo con lo manifestado voluntariamente por el padre de familia o acudiente durante el proceso de matrícula.

CUADRO 1. Resumen univariado de las variables de estudio

\begin{tabular}{|l|c|c|c|c|c|}
\hline \multirow{2}{*}{ Ciencias Naturales } & Mínimo & Máximo & \multicolumn{2}{|c|}{ Media } & Desviación estándar \\
\cline { 2 - 6 } & Estadístico & Estadístico & Estadístico & Error estándar & Estadístico \\
\hline Ciencias Sociales & 0.0 & 5.0 & 3.171 & .0199 & .8007 \\
\hline Educación Artística & 0.0 & 5.0 & 3.354 & .0170 & .6844 \\
\hline Educación ética & 0.0 & 5.0 & 3.645 & .0147 & .5932 \\
\hline Educación física & 0.0 & 5.0 & 3.585 & .0170 & .6861 \\
\hline Religión & 0.0 & 5.0 & 3.919 & .0171 & .6902 \\
\hline Lengua Castellana & 0.0 & 5.0 & 3.625 & .0137 & .5503 \\
\hline Inglés & 0.0 & 5.0 & 3.328 & .0151 & .6079 \\
\hline Tecnología e Informática & 0.0 & 5.0 & 3.236 & .0178 & .7146 \\
\hline Matemáticas & 0.0 & 5.0 & 3.488 & .0160 & .6424 \\
\hline Rendimiento académico global \\
(media de 10 asignaturas)
\end{tabular}

\begin{tabular}{|l|c|c|}
\hline \multirow{2}{*}{ Estrato } & 1 & $98.8 \%$ \\
\cline { 2 - 3 } & 2 & $1.2 \%$ \\
\hline \multirow{2}{*}{ Sexo } & Femenino & $44.4 \%$ \\
\cline { 2 - 3 } & Masculino & $55.6 \%$ \\
\hline
\end{tabular}

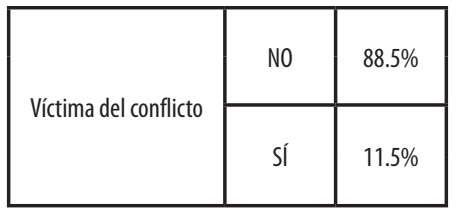

La escala de desempeńos definida por la institución educativa de acuerdo con el sistema de evaluación escolar enmarcado en el decreto 1290 de 2009 establece los siguientes niveles: Nivel bajo: 0.0-2.9; nivel básico: 3.0-3.7; nivel alto: 3.8-4.4 y nivel superior: 4.5-5.0.

Inicialmente, la tabla de datos involucraba la variable "lugar de procedencia"; sin embargo, la variable fue descartada por razones de tipo metodológico, por cuanto no aportaba información relevante para el proyecto "Representaciones sociales de los estudiantes de la IEAR acerca del postconflicto", en el cual se enmarca el presente estudio. 


\section{Procedimiento}

Posterior a la autorización por parte de la Rectoría de la IEAR, se contactó con el administrador del sistema de información para generar la consulta en MySQL con los datos requeridos, dado que los informes del software Academic Reports no contemplaban la información necesaria de manera condensada.

En segunda instancia, la información fue revisada por el grupo de investigadores del proyecto sobre el cual se aborda este estudio, quienes descartaron la variable "lugar de procedencia". Asimismo, se determinó que el análisis del rendimiento académico de los estudiantes se haría de acuerdo con el promedio de las calificaciones en cada una de las asignaturas básicas con los factores de influencia: el estrato socioeconómico, sexo y su contacto o no con el conflicto armado.

\section{Diseño y análisis estadístico}

Este estudio fue de tipo no experimental, transversal y correlacional, puesto que se identificaron las características del rendimiento académico de los estudiantes de IEAR, para luego estudiar las relaciones entre estas variables. Para lo anterior, se inició con un proceso descriptivo de las variables con base en su resumen univariado, las características de correlación entre la variable "asignaturas" y, finalmente, los procedimientos correlacionales entre variables.

Para el procesamiento y análisis de datos se utilizó el programa $\mathrm{R}$ y el paquete FactoMineR. Se realizaron análisis descriptivos y para el análisis correlacional de las variables se utilizó el coeficiente de correlación de Pearson.

\section{Resultados}

Una primera descripción de los datos, según su análisis univariado (ver cuadro 1), permitió establecer que la asignatura que presenta mejor resultados en el desempeño es educación física, con una media de 3.9, y la más baja matemáticas, con una media de 3.1. La escala institucional de desempeńo para cada caso es alto y básico, respectivamente. 
El estrato 1 es el más representativo de la población con un porcentaje del $98.8 \%$, aspecto que indica que los estudiantes de la IEAR pertenecen a familias de bajos recursos. Asimismo, se determinó que $55.6 \%$ de la población es de genero masculino.

En relación con el rendimiento académico, las calificaciones de los estudiantes en este análisis tienden a estar en el nivel de desempeño básico; incluso la media tiende a ubicarse ligeramente por debajo de 3.5 en la mayor parte de asignaturas, así como en la media del rendimiento global.

De esta manera, para determinar la posible asociación entre desempeño académico por asignatura, el contacto del estudiante con el conflicto armado, la edad, el sexo y el estrato, se llevó a cabo una correlación bilateral de Pearson entre todas estas variables.

Así, los resultados de la asociación de la variable asignaturas y sexo del estudiante se detallan en el cuadro 2.

Según los datos, existe una correlación positiva moderada entre las asignaturas: ciencias sociales, ciencias naturales, religión, lingüística y literatura, idioma extranjero y matemáticas. Esto implica que estas asignaturas contribuyen en mayor medida al rendimiento académico de los estudiantes, expresado en todas las calificaciones tenidas en consideración. Por su parte, las demás asignaturas consideran una correlación positiva baja.

Asimismo. no se encontró una relación significativa respecto a la variable sexo (hombres y mujeres); dicha relación no se ha encontrado en función del rendimiento académico cuyos niveles no se asocian a cambios significativos en las calificaciones; sin embargo, si se detallan los perfiles de cada asignatura, se puede verificar que tanto en hombres como en mujeres la asociación tiene el mismo valor, pero la correlación es mayormente positiva en mujeres y negativa en hombres, aspecto que señala que las mujeres se asocian en mayor medida al rendimiento académico. 


\begin{tabular}{|c|c|c|c|c|c|c|c|c|c|c|c|c|}
\hline 홀 & $\begin{array}{l}\text { * } \\
\text { o. } \\
\\
\end{array}$ & $\frac{w}{o}$ & $\bar{o}_{i}$ & 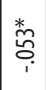 & ఫे & \begin{tabular}{l}
$*$ \\
\multirow{2}{0}{} \\
1 \\
1
\end{tabular} & 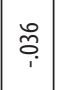 & \begin{tabular}{l}
$*$ \\
\multirow{2}{*}{} \\
\\
\\
\end{tabular} & 只 & \begin{tabular}{l|}
$*$ \\
\multirow{2}{5}{} \\
$i$ \\
\end{tabular} & 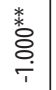 & \\
\hline 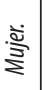 & 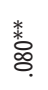 & $\frac{\frac{*}{*}}{\infty}$ & $\bar{\varnothing}$ & 䓠 & ষ্. & 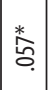 & ஜ̊. & 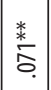 & 응 & 粘 & & 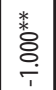 \\
\hline $\begin{array}{l}\dot{\bar{\Sigma}} \\
\stackrel{\Sigma}{\Sigma} \\
\Sigma\end{array}$ & $\stackrel{\text { 常 }}{\stackrel{f}{f}}$ & $\begin{array}{l}\underset{*}{*} \\
\stackrel{*}{+} \\
\stackrel{+}{+}\end{array}$ & $\begin{array}{l}\text { 类 } \\
\stackrel{\vartheta}{\vartheta}\end{array}$ & 䊑 & $\underset{m}{\stackrel{*}{*}}$ & \begin{tabular}{|l|}
$\stackrel{*}{*}$ \\
\multirow{f}{*}{.} \\
\end{tabular} & \begin{tabular}{l}
$\underset{*}{*}$ \\
\multirow{2}{f}{} \\
\end{tabular} & 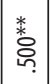 & 䊑 & & $\stackrel{*}{*}$ & 㐘 \\
\hline 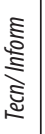 & 㐘 & $\begin{array}{l}\text { 粪 } \\
\text { m. }\end{array}$ & 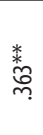 & $\stackrel{*}{\underset{n}{\approx}}$ & 莃 & 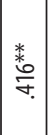 & $\mid \begin{array}{l}\text { 类 } \\
\text { 命 }\end{array}$ & 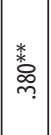 & & 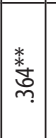 & 응 & 음 \\
\hline$\stackrel{\check{\Xi}}{\Xi}$ & $\stackrel{\text { * }}{\stackrel{*}{f}}$ & 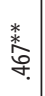 & $\underset{n}{\stackrel{*}{*}}$ & 糙 & 㐘 & \begin{tabular}{l}
$*$ \\
\multirow{2}{*}{} \\
$\stackrel{9}{q}$
\end{tabular} & $\underset{\underset{f}{*}}{\stackrel{*}{F}}$ & & 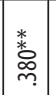 & 糔 & $\frac{*}{5}$ & 糔 \\
\hline 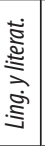 & $\stackrel{*}{\underset{\Im}{\Im}}$ & $\begin{array}{l}\text { 类 } \\
\text { 守. }\end{array}$ & 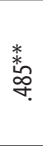 & $\begin{array}{l}\text { * } \\
\text { *o } \\
\text { 字 }\end{array}$ & $\begin{array}{l}\text { 类 } \\
\text { 今. }\end{array}$ & 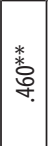 & & \begin{tabular}{|l|}
$\stackrel{*}{*}$ \\
$\stackrel{f}{f}$ \\
\end{tabular} & \begin{tabular}{|c} 
类 \\
$\stackrel{9}{m}$ \\
\end{tabular} & \begin{tabular}{|l} 
\\
\multirow{2}{*}{} \\
$\sigma$ \\
$\sigma$
\end{tabular} & ஜூ & $\begin{array}{l}0 \\
0 \\
i \\
i\end{array}$ \\
\hline 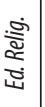 & $\begin{array}{l}\text { 絭 } \\
\text { q⿱龴⿱乛亅㇒. }\end{array}$ & 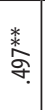 & 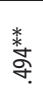 & 㐘 & $\begin{array}{l}\text { 䊅 } \\
\stackrel{f}{\sigma}\end{array}$ & & \begin{tabular}{l} 
类 \\
0 \\
\hdashline
\end{tabular} & 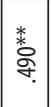 & 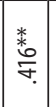 & $\begin{array}{l}\text { 粚 } \\
\stackrel{f}{f}\end{array}$ & \begin{tabular}{l}
$*$ \\
\multirow{2}{*}{} \\
0
\end{tabular} & 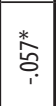 \\
\hline 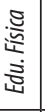 & $\stackrel{*}{\stackrel{*}{y}}$ & 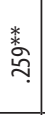 & $\begin{array}{l}\text { 类 } \\
\text { స్ }\end{array}$ & 絭 & & 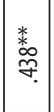 & $\begin{array}{l}\text { 类 } \\
\text { 今े }\end{array}$ & 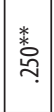 & $\begin{array}{l}\text { 絭 } \\
\text { ఫે }\end{array}$ & 粪 & ఫ़ & षे \\
\hline 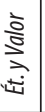 & 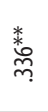 & 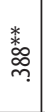 & 㐘 & & $\begin{array}{l}\text { 絭 } \\
\text { 品 }\end{array}$ & 糔 & $\begin{array}{l}\text { 粪 } \\
\text { ơ }\end{array}$ & 糔 & 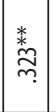 & 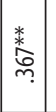 & $\stackrel{*}{2}$ & 䇾 \\
\hline 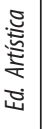 & $\underset{⿱}{*}$ & $\begin{array}{l}\frac{*}{*} \\
\frac{8}{q}\end{array}$ & & 㐘 & $\begin{array}{l}\text { 㐘 } \\
\text { N }\end{array}$ & 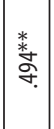 & 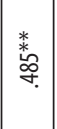 & 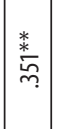 & \begin{tabular}{|l|}
$*$ \\
\multirow{2}{*}{} \\
$\tilde{m}$
\end{tabular} & 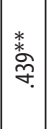 & $\bar{\phi}$ & $\overline{\tilde{o}}$ \\
\hline 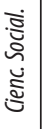 & 楚 & & $\frac{*}{\frac{*}{q}}$ & $\begin{array}{l}\text { * } \\
\underset{\infty}{\infty} \\
\infty \\
m\end{array}$ & $\begin{array}{l}\text { 㐘 } \\
\stackrel{\sigma}{*}\end{array}$ & $\begin{array}{l}\underset{*}{*} \\
\underset{\sigma}{\sigma} . \\
\end{array}$ & $\begin{array}{l}\underset{*}{*} \\
\stackrel{\sigma}{\sigma} .\end{array}$ & 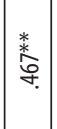 & \begin{tabular}{l} 
委 \\
\multirow{w}{*}{} \\
$m$
\end{tabular} & 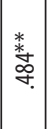 & $\begin{array}{l}\frac{*}{*} \\
\stackrel{0}{o}\end{array}$ & $\begin{array}{l}* \\
\\
0 \\
\end{array}$ \\
\hline 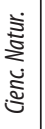 & & $\frac{*}{\frac{*}{6}}$ & 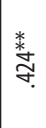 & $\underset{m}{*}$ & $\underset{⿱}{\stackrel{*}{y}}$ & $\begin{array}{l}\text { 絭 } \\
\stackrel{q}{q} . \\
\end{array}$ & $\stackrel{*}{\underset{\Im}{\Im}}$ & 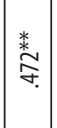 & $\begin{array}{l}* \\
\text { *o } \\
\stackrel{m}{m}\end{array}$ & 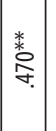 & $\begin{array}{l}* \\
\stackrel{*}{0} \\
\stackrel{0}{0}\end{array}$ & $\begin{array}{l}* \\
\text { * } \\
0 \\
0 \\
0\end{array}$ \\
\hline & 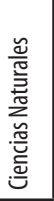 & 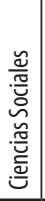 & 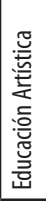 & 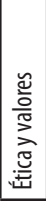 & 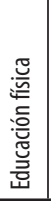 & 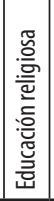 & 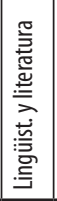 & 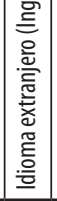 & 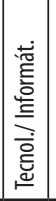 & 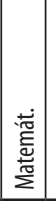 & 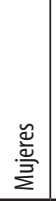 & 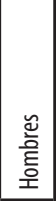 \\
\hline
\end{tabular}


Ahora bien. la contribución de las variables: víctimas del conflicto, estrato, sexo y edad, al rendimiento académico, se puede ver en el cuadro 3 de correlaciones:

CUADR0 3. Correlación entre asignaturas básicas y variables víctimas de conflicto, edad y estrato

\begin{tabular}{|l|c|c|c|c|c|}
\hline & \multirow{2}{*}{ Vict. Conflicto } & \multirow{2}{*}{ Estrato } & \multirow{2}{*}{ Edad } & \multicolumn{2}{|c|}{ Sexo } \\
\cline { 5 - 6 } & & & & Femenino & Masculino \\
\hline Ciencias Naturales & $-0.062^{*}$ & -0.045 & $-0.429^{* *}$ & $.080^{* *}$ & $-.080^{* *}$ \\
\hline Ciencias Sociales & -0.026 & 0.002 & $-0.360^{* *}$ & $.081^{* *}$ & $-.081^{* *}$ \\
\hline Educación Artística & -0.007 & 0.026 & $-0.251^{* *}$ & .031 & -.031 \\
\hline Ética y valores & -0.031 & -0.038 & $-0.154^{* *}$ & $.053^{*}$ & $-.053^{*}$ \\
\hline Educación física & 0.026 & 0.036 & $-.090^{* *}$ & -.004 & .004 \\
\hline Educación religiosa & -0.003 & -0.006 & $-0.384^{* *}$ & $.057^{*}$ & $-.057^{*}$ \\
\hline Lingǘstica y literatura & -0.039 & 0.006 & $-0.122^{* *}$ & .036 & -.036 \\
\hline Idioma extranjero (Inglés) & -0.044 & $-0.057^{*}$ & $-0.408^{* *}$ & $.071^{* *}$ & $-.071^{* *}$ \\
\hline Tecnología e Informática & $-0.076^{* *}$ & -0.039 & $-0.260^{* *}$ & .010 & -.010 \\
\hline Matemáticas & -0.013 & -0.029 & $-0.351^{* *}$ & $.051^{*}$ & $-.051^{*}$ \\
\hline Edad & $0.062^{*}$ & $0.064^{* *}$ & & $-.077^{* *}$ & $.077^{* *}$ \\
\hline Estrato & $0.056^{*}$ & & $0.064^{* *}$ & -.005 & .005 \\
\hline Hombres & .015 & .005 & $.077^{* *}$ & $-1.000^{* *}$ & \\
\hline Mujeres & -.015 & -.005 & $-.077^{* *}$ & & $-1.000^{* *}$ \\
\hline
\end{tabular}

**. La correlación es significativa en el nivel 0,01 (2 colas).

* . La correlación es significativa en el nivel 0,05 (2 colas).

El cuadro 3 muestra como resultado del análisis de la relación entre asignaturas y edad, que existe correlación negativa muy baja o baja. En este sentido, no se puede considerar que la edad sea un factor que determine el desempeño académico. De igual manera, no se encontró relación significativa entre el rendimiento académico, el estrato y el sexo del estudiante.

Asimismo, para determinar la incidencia de la variable conflicto armado sobre los sujetos objeto de estudio, se realizaron dos Rplots (gráficas 1 y 2) para visualizar gráficamente el comportamiento de los sujetos en función del rendimiento académico.

Por otra parte y, en comparación con la gráfica 1, la gráfica 2 establece el comportamiento de los sujetos que han sido víctimas del conflicto armado. 
GRÁFICA 1. Comportamiento rendimiento académico de estudiantes IEAR

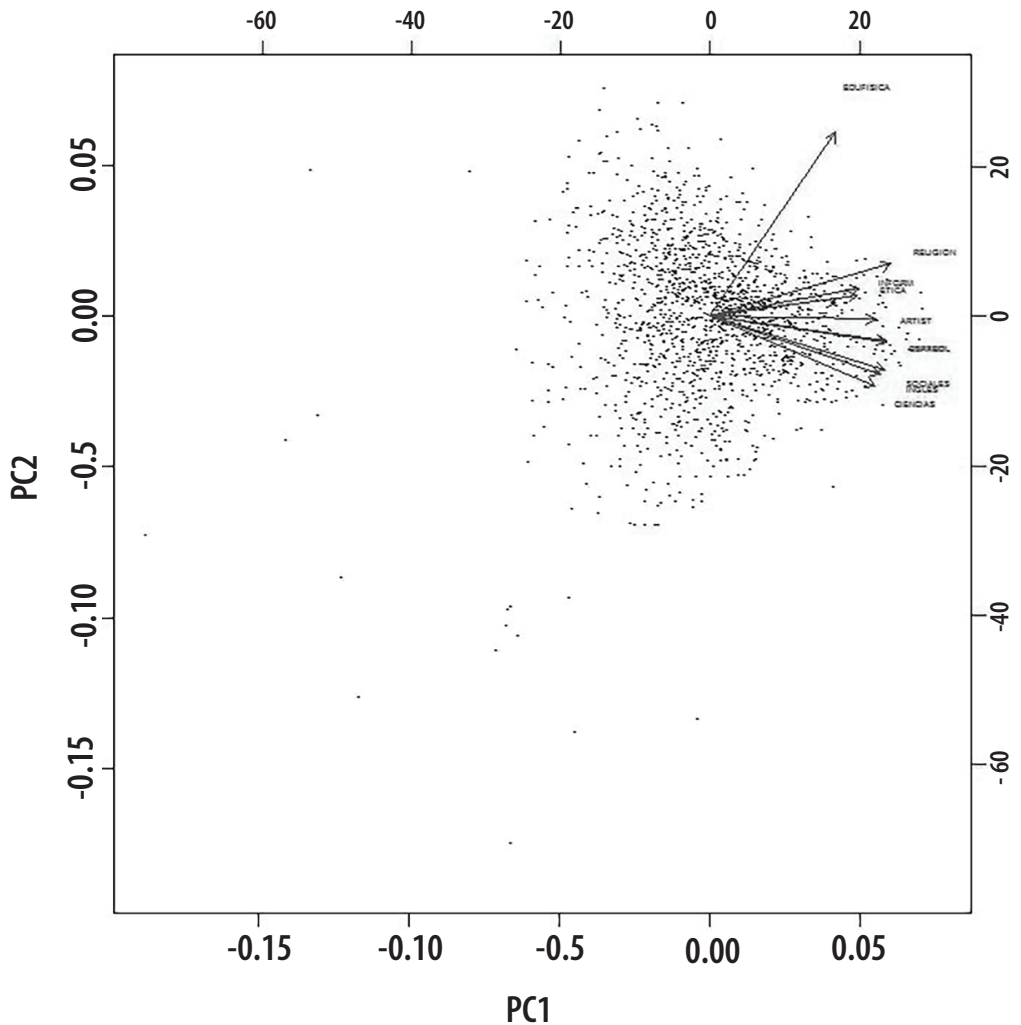

En la primera gráfica se evidencia que la asignatura educación física es la que tiene, en general, un comportamiento más diferenciado frente a las otras, dado que en ella se ubican los estudiantes con mejores calificaciones. Enseguida se encuentran las áreas de religión y ética.

Los sujetos ubicados en el lado izquierdo del cuadrante representan los estudiantes con desempeño bajo, mientras los de la derecha, aquellos con desempeño básico, alto y superior. Se puede observar, igualmente, que los vectores de asignaturas se ubican, en su totalidad, en valores de desempeños por encima del básico. 
GRÁFICA 2. Diferencias en rendimiento académico de estudiantes víctimas del conflicto armado.

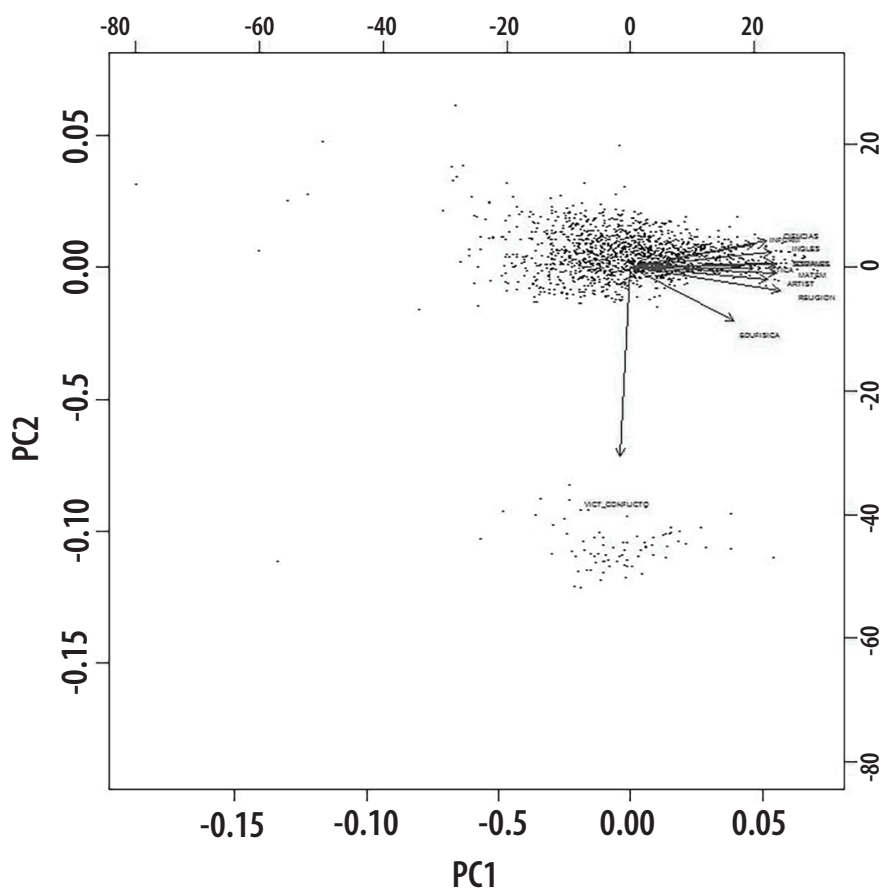

La gráfica permite visualizar que los estudiantes víctimas del conflicto presentan una gran diferenciación con aquellos que no han sufrido esta situación. Se presentan dos grupos muy diferenciados. Igualmente, se evidencia en la dimensión 1 (PC1) que estos sujetos presentan correlación del orden de -0.05 en relación con las asignaturas, aspecto que obliga a "desplazar" los vectores de asignaturas hacia desempeños más bajos.

Adicionalmente, se llevó a cabo una prueba de diferencia de medias del rendimiento académico por asignaturas y la variable víctima del conflicto. Los datos, sometidos a la prueba de Levene de calidad de varianzas se detallan en el cuadro 4. Así, los datos arrojaron que sí existe compatibilidad entre la hipótesis de igualdad de la media poblacional por asignaturas y las diferencias entre las medias de grupos representados como víctimas o no del conflicto armado. 


\section{CUADRO 4. Diferencia de medias asignaturas básicas y grupos víctimas o no de conflicto armado}

\begin{tabular}{|c|c|c|c|c|c|c|c|c|c|}
\hline & \multicolumn{2}{|c|}{$\begin{array}{l}\text { Prueba de } \\
\text { Levene calidad } \\
\text { varianzas }\end{array}$} & \multicolumn{7}{|c|}{ prueba t para la igualdad de medias } \\
\hline & \multirow[t]{2}{*}{$F$} & \multirow[t]{2}{*}{ Sig. } & \multirow[t]{2}{*}{$t$} & \multirow[t]{2}{*}{$g l$} & \multirow[t]{2}{*}{$\begin{array}{c}\text { Sig. } \\
\text { (bilateral) }\end{array}$} & \multirow[t]{2}{*}{$\begin{array}{l}\text { Diferencia } \\
\text { de medias }\end{array}$} & \multirow{2}{*}{$\begin{array}{c}\text { Diferencia } \\
\text { error } \\
\text { estándar }\end{array}$} & \multicolumn{2}{|c|}{$\begin{array}{c}\text { 95\% de intervalo } \\
\text { de confianza de la } \\
\text { diferencia }\end{array}$} \\
\hline & & & & & & & & Inferior & Superior \\
\hline $\begin{array}{l}\text { Ciencias } \\
\text { Naturales }\end{array}$ & .207 & .649 & 2.486 & 1618 & .013 & .2307 & .0928 & .0487 & .4127 \\
\hline $\begin{array}{l}\text { Ciencias } \\
\text { Sociales }\end{array}$ & 2.131 & .145 & 1.050 & 1618 & .294 & .0834 & .0794 & -.0724 & .2392 \\
\hline $\begin{array}{l}\text { Educación } \\
\text { Artística }\end{array}$ & 4.293 & .038 & .295 & 1618 & .768 & .0203 & .0689 & -.1147 & .1554 \\
\hline Ética y valores & .002 & .969 & 1.262 & 1618 & .207 & .1005 & .0796 & -.0557 & .2566 \\
\hline $\begin{array}{l}\text { Educación } \\
\text { física }\end{array}$ & 1.234 & .267 & -1.058 & 1618 & .290 & -.0848 & .0801 & -.2419 & .0723 \\
\hline $\begin{array}{l}\text { Educación } \\
\text { religiosa }\end{array}$ & 2.222 & .136 & .139 & 1618 & .889 & .0089 & .0639 & -.1164 & .1342 \\
\hline $\begin{array}{l}\text { Lingüística y } \\
\text { literatura }\end{array}$ & .308 & .579 & 1.550 & 1618 & .121 & 1093. & 年. & -.0290 & .2476 \\
\hline $\begin{array}{l}\text { Idioma } \\
\text { extranjero } \\
\text { (Inglés) }\end{array}$ & 4.916 & .027 & 1.769 & 1618 & .077 & .1466 & .0829 & -.0159 & .3092 \\
\hline $\begin{array}{l}\text { Tecnología e } \\
\text { Informática }\end{array}$ & .106 & .744 & 3.079 & 1618 & .002 & .2290 & .0744 & .0831 & .3748 \\
\hline Matemáticas & 1.170 & .279 & .533 & 1618 & .594 & .0497 & .0933 & -.1333 & .2328 \\
\hline
\end{tabular}

Los límites de intervalo de confianza para la diferencia indican que, para todas las asignaturas, excepto tecnología e informática y ciencias naturales, los límites inferiores representan valores negativos y los valores superiores, valores positivos. El hecho de que el valor 0 no esté incluido dentro del intervalo de confianza de la diferencia, también indica que se puede aceptar la hipótesis de igualdad de medias por asignaturas.

Por otra parte, los resultados del cuadro 5 de estadísticos, indican que la media del rendimiento académico por asignaturas se ve afectada por la exposición de estudiantes al conflicto armado. 
RENDIMIENTO ACADÉMICO DE ESTUDIANTES DE SECUNDARIA SEGÚN ASIGNATURAS...

TABLA 5. Estadístico de asignaturas en función de variable conflicto armado

\begin{tabular}{|c|c|c|c|c|}
\hline Asignatura & $\begin{array}{c}\text { Vict. Conflicto } \\
\text { Armado } \\
\text { Si }(N=1434) \text { No } \\
(N=186)\end{array}$ & Media & Desviación estándar & Media de error estándar \\
\hline \multirow{2}{*}{ Ciencias Naturales } & No & 3.182 & .7980 & .0203 \\
\hline & $\mathrm{Si}$ & 2.951 & .8288 & .0938 \\
\hline \multirow{2}{*}{ Ciencias Sociales } & No & 3.358 & .6851 & .0174 \\
\hline & $\mathrm{Si}$ & 3.274 & .6706 & .0759 \\
\hline \multirow{2}{*}{ Educación Artística } & No & 3.646 & .5934 & .0151 \\
\hline & $\mathrm{Si}$ & 3.626 & .5925 & .0671 \\
\hline \multirow{2}{*}{ Ética y valores } & No & 3.590 & .6833 & .0174 \\
\hline & $\mathrm{Si}$ & 3.490 & .7381 & .0836 \\
\hline \multirow{2}{*}{ Educación física } & No & 3.915 & .6928 & .0176 \\
\hline & $\mathrm{Si}$ & 4.000 & .6345 & .0718 \\
\hline \multirow{2}{*}{ Educación religiosa } & No & 3.626 & .5543 & .0141 \\
\hline & $\mathrm{Si}$ & 3.617 & .4661 & .0528 \\
\hline \multirow{2}{*}{ Lingüística y literatura } & No & 3.334 & .6058 & .0154 \\
\hline & $\mathrm{Si}$ & 3.224 & .6437 & .0729 \\
\hline \multirow{2}{*}{ Idioma extranjero (Inglés) } & No & 3.243 & .7180 & .0183 \\
\hline & $\mathrm{Si}$ & 3.096 & .6317 & .0715 \\
\hline \multirow{2}{*}{ Tecnología e Informática } & No & 3.499 & .6378 & .0162 \\
\hline & $\mathrm{Si}$ & 3.271 & .6958 & .0788 \\
\hline \multirow{2}{*}{ Matemáticas } & No & 3.104 & .8071 & .0206 \\
\hline & $\mathrm{Si}$ & 3.054 & .7436 & .0842 \\
\hline
\end{tabular}

De esta manera, se denota que los estudiantes que han sido víctimas del conflicto armado presentan una media más baja que los estudiantes que no lo han sido.

\section{Discusión}

El rendimiento académico puede estar influido por múltiples variables como la motivación, inteligencia y aptitudes, autoconcepto, hábitos, estrategias y estilos de aprendizaje, hábitos de estudio, aspectos familiares, etc. (González, 2003; Gómez, 2003; Reinoso, 2011). En este estudio se ha llevado a cabo, principalmente, un análisis de una variable de carácter social acorde con la problemática colombiana: el contacto con el conflicto armado, con el fin de conocer en qué medida pueden asociarse. 
De esta manera, para dar respuesta al primer objetivo, los datos de este estudio indican que la población corresponde a un conjunto de jóvenes con un desempeño académico básico, dado que así lo demuestran las puntuaciones obtenidas al tenor del criterio de calificaciones por asignaturas básicas. La mayor parte de los resultados ubican a los sujetos de estudio en rangos medios de normalidad en el desempeño por asignaturas; sin embargo, establece diferencias en aquellos en contacto con el conflicto armado. Respecto al rendimiento académico obtenido con la media de las asignaturas básicas establecidas por la Ley general de Educación de Colombia, los sujetos muestran un nivel de desempeño básico, en torno a una calificación por debajo de 3.5. La asignatura con mejores desempeños es educación física.

Los resultados del análisis de correlación entre asignaturas indican una relación positiva muy baja entre rendimiento académico y el sexo del estudiante, entre 0.01 y 0.08 para mujeres y -1.0 y 0.08 para hombres. Esto implica que en la IEAR, aunque existe correlación, el género se asocia a cambios poco significativos en las calificaciones. En la misma vía del análisis estadístico de este estudio, Echavarri, Godoy y Olaz (2007) establecen que sí existen diferencias estadísticamente significativas en el rendimiento académico de hombres y mujeres universitarios, dando como resultado en su investigación que las correlaciones entre las combinatorias de los tests y el rendimiento académico se presentan como moderadas y grandes. En el ambito universitario, las diferencias de género en aptitudes cognitivas y la relación de estas con el rendimiento académico, sugieren la importancia de considerar estrategias pedagógicas enfocadas a estimular diferencialmente las habilidades cognitivas de hombres y mujeres, puesto que cada sexo emplea estrategias diferentes para resolver problemas complejos; según los investigadores, los hombres hacen más uso del razonamiento abstracto y espacial, mientras que las mujeres recurren con mayor frecuencia a estrategias verbales. En este sentido, se pueden establecer diferencias entre la postura de los investigadores y este estudio frente a las diferencias significativas entre género y rendimiento académico, debido al nivel de maduración cognitiva de los estudiantes de educación básica y media.

Ahora bien, en atención al segundo objetivo planteado en el presente estudio, se estableció, a partir de la correlación entre 
variables, que el rendimiento académico de los estudiantes de la IEAR se vió influido por el contacto con el conflicto armado, aspecto que ratifica lo mencionado por la UNESCO (2011) en razón a que los conflictos armados siguen representando un importante obstáculo para el desarrollo humano en muchas partes del mundo.

Los resultados indicaron una relación significativa de tipo negativo entre las dos variables. Esta relación presentó claramente que los estudiantes que sufrieron o percibieron hechos violentos de manera personal o indirecta, obtuvieron una calificación media en su rendimiento académico más baja. En esta vía, Hewitt, N., Gantiva, C. A., Vera, A. et al. (2014) argumentan que niños y adolescentes expuestos al conflicto armado presentan afectaciones psicológicas que inciden directamente en la salud mental y, por consiguiente, en el desempeño escolar. Asimismo, otras investigaciones coinciden con estos resultados, argumentando que el conflicto armado podría ser un factor de riesgo para el bajo rendimiento académico de los estudiantes, debido a la condición de desplazamiento, en razón a que la atención a los desplazados en los centros educativos puede tardar varios meses (Quintero, 2014).

El análisis de correlación de estas dos variables estableció que existe una menor asociación negativa del rendimiento académico específico en las asignaturas educación física, educación artística y religión. En esta perspectiva, el Comité Internacional de la Cruz Roja (2011) y algunos autores (Infante, 2011; Perdomo, 2014) sostinen que el deporte, la música, la danza y actividades lúdicas promueven la resiliencia de jóvenes afectados por los conflictos armados y otras situaciones de violencia. En este sentido, se puede determinar que las asignaturas que ofrecen actividades que generan sentimientos y emociones en los estudiantes permiten obtener mejores resultados en el desempeńo escolar dedibo a la motivación.

Igualmente, se encontró una relación significativa de tipo positivo entre estrato y conflicto armado, dado que los estudiantes de estrato 1 son los más afectados por este flagelo; en contravía, los estudiantes de estrato 2 de la IEAR (aunque pocos en la población objeto de estudio) no están involucrados en ambientes relacionados con el conflicto armado. En este sentido, Bohada (2010) y Posada et al. (2009) han argumentado que las poblaciones de estratos bajos son más susceptibles de enfrentar síntomas de conflicto armado que los estratos altos. 
Respecto a las posibles limitaciones de este estudio, cabría mencionar que han quedado por fuera del planteamiento variables relevantes asociadas al conflicto armado que esta investigación considera se deben tener en cuenta, tales como nivel de ansiedad, depresión, agresión u otros aspectos de tipo actitudinal.

Para concluir y, en referencia a las aplicaciones prácticas de este estudio, sería conveniente que durante el proceso de enseñanza se tengan en cuenta aspectos didácticos y que generen motivación en el estudiante, puesto que la motivación guarda relación significativa con el buen rendimiento académico. De esta manera, parece factible pensar que un estudiante expuesto a actividades de motivación en la escuela, a pesar de haber vivido situaciones de conflicto armado, pueden obtener mejoras sustanciales en los resultados académicos.

\section{Referencias bibliográficas}

Avila, Jonh, Quiñonez, Fanny Milena; Sánchez, Henry; Hidalgo, José; Palomino, Marcela Andrea; Sandino, Andrea y Delgado, Heriberto. "El informe PISA 2012: Campaña publicitaria contra la educación pública”, en Revista Educación y Cultura, 102, 2014.

Bohada, María del Pilar. "Desplazamiento forzado y condiciones de vida de las comunidades de destino: el caso de Pasto. Nariño", en Revista de Economía Institucional, 23(12), 2010, pp. 259-298. Disponible en: http://www.economiainstitucional.com/pdf/No23/mbohada23.pdf. Fecha de acceso, 20 de junio de 2015.

Comité Internacional de la Cruz Roja. "Los niños afectados por los conflictos armados y otras situaciones de violencia. Informe de taller. Comité Internacional de la Cruz Roja, Ginebra, 2011. Disponible en: https://www.icrc.org/spa/ assets/files/publications/icrc-003-4082.pdf. Fecha de acceso 20 de junio de 2015.

Cruz. Feernando. "Educar para gestionar conflictos en una sociedad fragmentada". Tesis doctoral, Departamento de Teoría e Historia de la Educación, Universidad de Barcelona, Barcelona, 2000. Disponible en: http://diposit.ub.edu/dspace/ handle/2445/43079. Fecha de acceso 15 de julio de 2015. 
Echavarri, Maximilinao; Godoy, Juan Carlos; y Olaz, Fabían. "Diferencias de género en habilidades cognitivas y rendimiento académico en estudiantes universitarios", en Revista Universitas Psychologica, 6(2), 2007, pp. 319-329

Gómez, Margarita. "Algunos factores que influyen en el éxito académico de los estudiantes universitarios". Tesis doctoral, Departamento de didáctica de las ciencias y las matemáticas, Universidad Autónoma de Barcelona, Bellaterra, 2003. Disponible en:http://www.raco.cat/index.php/ensenanza/article/ viewFile/87862/216396. Fecha de acceso 20 de julio de 2015.

González, Coral. "Factores determinantes del bajo rendimiento académico en educación secundaria”. Tesis doctoral, Departamento de Métodos de investigación y diagnóstico en educación, Universidad Complutense de Madrid, Madrid, 2003. Disponible en: http://biblioteca.ucm.es/tesis/edu/ ucm-t27044.pdf. Fecha de acceso, 22 de junio de 2015.

Infante, Armando. "El papel de la educación en situaciones de postconflicto: estrategias y recomendaciones", en Revista Hallazgos, 11(21), 2011, pp. 223-245. Disponible en: http://revistas.usta.edu.co/index.php/hallazgos/article/ view/1156. Fecha de acceso, 19 de junio de 2015.

Martínez, Boom Alberto. "De la escuela expansiva a la escuela competitiva: dos modos de modernización educativa en América Latina", Anthropos, Bogotá, 2004. Disponible en: http://www.albertomartinezboom.com/Comentarios/2005_Tesis\%20doctoral_Catalina_Olga_Maya.pdf. Fecha de acceso, 1 de julio de 2015.

Organización Médicos Sin Fronteras-MSF. "Tres veces víctimas. Conflicto armado y salud mental en el departamento del Caquetá", 2009. Disponible en: http://onsm.ces.edu.co/uploads/ files/1143745_Informe-salud-mental-CAQUETA--Medicossin-fronteras.pdf. Fecha de acceso, 12 de julio de 2015.

Perdomo, Orlando. "Resiliencia en la enseñanza musical en población infantil y juvenil en situación de desplazamiento forzado", en Revista Amazonia Investiga, 3 (5), 2014, pp. 5-33. Disponible en:http://www.udla.edu.co/revistas/ index.php/amazonia-investiga/article/view/50. Fecha de acceso, 20 de julio de 2015.

Posada, María Carmenza; Rodríguez, Alfonso; Vega, Gerardo y Balbin, Jesús. "Víctimas, violencia y despojo. Informe de la in- 
vestigación acerca de víctimas del conflicto armado", 2009. Disponible en http://web.usbmed.edu.co/usbmed/formacion/docs/victimas.pdf. Fecha de acceso, 20 de julio de 2015.

Quintero, López. "La educación como blanco de ataque: Mirada sobre las consecuencias del conflicto armado en Colombia”, 2014. Disponible en: http://centrovirtual.idep.edu. co/index.php/component/k2/item/39-agosto. Fecha de acceso, 11 de julio de 2015.

Hewitt, Nohelia; Gantiva, Carlos Andrés; Vera, Anderssen; Cuervo, Mónica Paulina; Hernández, Nelly Lillliam; Juárez, Fernando y Parada, Arturo José. "Afectaciones psicológicas de niños y adolescentes expuestos al conflicto armado en una zona rural de Colombia", en Acta Colombiana de Psicología, 17(1), 2014, pp. 79-89, doi: 10.14718/ACP.2014.17.1.9. de 2015. Disponible en: http://www.scielo.org.co/scielo. php?script=sci_arttext\&pid=S0123-91552014000100009. Fecha de acceso, 25 de julio

Reinoso, Elsa Laura. "Factores que determinan el rendimiento escolar en el nivel secundario en el Estado de Nuevo León”, 2011. Tesis doctoral, Instituto de investigaciones sociales, Universidad Autónoma de Nuevo León. Disponible en: http://dspace.leon.uia.mx:8080/xmlui/handle/123456789/96342. Fecha de acceso, 12 de julio de 2015.

Tarabini-Castellani, Aina. "Educación, pobreza y desarrollo: agendas globales, políticas nacionales, realidades locales", 2008. Tesis doctoral. Disponible en: http://www.tdx.cat/ bitstream/handle/10803/5138/atc1de1.pdf;jsessionid=FD 3E4B8DC5825F5CD0C965EAC4314615.tdx1 ? sequence $=1$. Fecha de acceso, 12 de julio de 2015.

Sánchez, Fabio y Díaz, Ana María. "Los efectos del conflicto armado en el desarrollo social colombiano, 1990-2002", 2005. Disponible en:https://economia.uniandes.edu.co/ assets/archivos/Documentos_CEDE/d2005-58.pdf. Fecha de acceso, 10 de julio de 2015.

Soberanis, Hoyos. Filosofía de la Educación, Madrid, Trotta, 2008. UNESCO. "Una crisis encubierta. Conflicto armado y educación. Informe de seguimiento de la EPT en el mundo", París, UNESCO, 2011. Disponible en: http://unesdoc.unesco.org/ images/0019/001921/192155S.pdf. Fecha de acceso, 12 de Julio de 2015. 